\title{
ACCESSIBILITY OF THE LOWEST QUINTET STATE OF ORGANIC MOLECULES THROUGH TRIPLET-TRIPLET ANNIHILATION; AN INDO CI STUDY
}

\author{
Bernhard DICK : \\ Lehrstuhl für Theoretische Chemie. Unicersität zu Köln. Greinstrasse 4. D-5000 Cologne HI. FRG \\ and \\ Bernhard NICKEL \\ Max-Planck-Institut für Biophysikalische Chemie, Am Fassherg. D-.3400 Göningen. FRG
}

Received 19 January 1983

By the spin-allowed annihilation of two metastable triplet states (triplet-triplet annihilation - TTA) one electronic ground state $\left(S_{0}\right)$ and one electronically excited singlet $\left(S_{l}\right)$ or triplet $\left(T_{j}\right)$ or quintet $\left(Q_{h}\right)$ state are created. provided the sum of :he excitation energies of the two metastable triplet states is sufficient for the creation of the particular excited state. On the bisis of semi-empirical calculations of the excitation energies of $T_{1}^{\prime}$ and $Q_{1}$ of forty-six conjugated organic compounds it is shown that $Q_{1}$ of benzene and some other compounds should be accessible through annihilation of like triplets (homo-TTA). and that $Q_{1}$ of many compounds should be accessible through annihilation of unlike triplets (hetero-TTA). The population of $Q_{1}$. competing with that of $S_{1}$. should cause an unusual magnetic-field dependence of the delayed nuorescence $S_{1} \rightarrow S_{13}$. In favourable cases, the population of $Q_{1}$ should lead to an inverse (positive) magnetic high-field effect on the delayed fluorescence.

\section{Introduction}

Organic molecules with an even number of electrons usually have singlet electronic ground states $\left(\mathrm{S}_{0}\right)$ and electronically excited states of singlet. triplet. quintet,... multiplicity. Nearly nothing is known on electronically excited states with a multiplicity higher than triplet. In particular. in no case is a lowest electronically excited quintet state $\left(Q_{1}\right)$ known (molecules with a quintet electronic ground state $Q_{0}$ are known [1-4]. but we do not refer to such molecules in the present paper). This lack of knowledge is a consequence of the fact that $Q_{1}$ always lies above the lowest excited singlet state $\left(S_{1}\right)$ and above the lowest excited triplet state $\left(T_{1}\right)$. These facts imply that the efficiency of intersystem crossing $S_{i} \leadsto T_{j} \rightarrow Q_{1}$ must be very low

\footnotetext{
* Present address: Department of Chemistry/D5. University of Pennsylvania. Philadelphia, PA 19104. USA.
}

because of the competing and in general very fast internal conversions of the upper excited states $S_{\text {, }}$ and $T_{j}$ into $S_{1}$ and $T_{1}$. respectively (Kasha's rule). Hence. compared to the population of $S_{1}$ and $T_{1}$ by optical excitation. no general and efficient method for the population of $Q_{1}$ is available. In contrast to $T_{1} \cdot Q_{1}$ in general cannot be metastable because of intersystem crossing $Q_{1} \rightarrow T_{h}$ to lower-lying triplet states $T_{k}$. Hence detection methods that depend on the metastability of the excited state to detect. cannot be used for the detection of $Q_{1}$. In particular. it should be very difficult to detect $Q_{1}$ of any compound by the phosphorescence $Q_{1} \rightarrow S_{0}$ or by electron spin resonance.

A spin-allowed process which in energetically favourable cases can lead to $Q_{1}$ is triplet-triplet annihilation (TTA). A pair of interacting molecules in their lowest and metastable triplet states $T_{1}$ and $T_{1}^{\prime}$ may have singlet. triplet and quintet 
character [5]. Therefore, the creation of excited singlet states $S_{1}$ or $S_{j}^{\prime}$ or excited triplet states $T_{k}$ or $T_{j}^{\prime}$ or excited quintet states $\mathbf{Q}_{m}$ or $\mathbf{Q}_{n}^{\prime}$ by TTA,

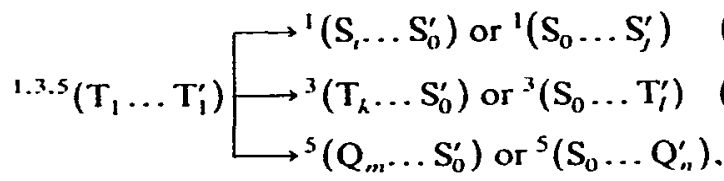

is spin allowed and will take place. provided the excitation energy of the particular excited state does not exceed the available excitation energy $E\left(T_{1}\right)+E\left(T_{1}^{\prime}\right)$. The TTA of like molecules $\left(T_{1}=\right.$ $T_{1}^{\prime}$ ) is called homo-TTA. and TTA of unlike molecules $\left(T_{1} \neq T_{1}^{\prime}\right)$ is called hetero-TTA. In the present paper. the simple term TTA will either mean homo-TTA or imply that the distinction between homo-TTA and hetero-TTA is not essential in the particular context.

The singlet reaction channel (1a) of TTA can be monitored directly through observation of the socalled delayed fluorescence $S_{1} \rightarrow S_{0}$ [6]. If in the primary process an upper excited singlet state $S_{i}$ $(i>1)$ is populated, also a delayed fluorescence $S_{1} \rightarrow S_{0}$ can be observed [7]. which. however, in general is very weak because of very fast internal conversion $S_{1} \rightarrow S_{1}$. The existence of at least one additional reaction channel can be inferred from the fact that the probability of creation of one excited singlet state by the annihilation of two triplet states is in general less than unity [8-13]. Of the two remaining TTA channels, the triplet channel (lb) must always contribute to the total TTA rate because there are always triplet states accessible by TTA. Indirect information on the possible contribution of the quintet channel (lc) to the total TTA rate can be obtained from the magnetic-field dependence of the delayed fluorescence [5,14-24] (see section 3). There is no doubt that in anthracene single crystals, that is, in the most thoroughly investigated system $[14,15,24], Q_{1}$ is not accessible through TTA.

The question, whether the lowest excited quintet state of any organic molecule can be populated by homo-TTA, has been answered differently. Sternlicht et al. [25] concluded from the results of a theoretical calculation of Parr et al. [26] that $Q_{1}$ of benzene $\left({ }^{5} A_{1 g}\right)$ is a possible final state of TTA. Suna [15] stated: "In practice, we shall always neglect $\lambda_{\mathrm{Q}}$ (that is, the quintet channel; see section $3)$, as there is no known system where double the triplet energy even remotely approaches the estimated energy of the lowest quintet state". Lendi et al. [19] concluded from the observed magnetic-field dependence of the delayed fluorescence from liquid solutions of pyrene. 1,2-benzanthracene. phenanthrene, and 3,4-benzpyrene [27] that with these compounds the energy of the lowest quintet state is smaller than twice the triplet energy. The only aromatic compound for which we found calculated quintet energies in the literature. is benzene $[26,28,29]$. If one assumes that the ab initio calculations of Peyerimhoff and Buenker [29] yield the correct order of states also for states of different multiplicity. then $\mathrm{Q}_{1}\left({ }^{5} \mathrm{~A}_{\mathrm{lg}}\right)$ of benzene lies below the lowest ' $E_{14}$ state. The excitation energy of the lowest ${ }^{1} E_{l u}$ state, however, is less than twice the triplet energy. Hence at least the case of benzene contradicts Suna's statement. Wirz [30] concluded from simple HMO calculations that, apart from benzene, the lowest quintet state of other aromatic compounds (e.g. of triphenylene, pentaphene, 1.2.3,4,5,6,7,8-tetrabenzanthracene) also might be accessible by TTA.

The present investigation is an attempt to achieve some progress in the quintet-TTA problem. First. we describe briefly a semi-empirical calculational procedure, which allows to calculate singlet states and triplet states with a single set of parameters. Second, from the satisfactory agreement of calculated and experimental excitation energies of $S_{1}$ and $T_{1}$ for a variety of compounds we shall conclude that our method yields the excitation energy of $Q_{1}$ with about the same accuracy as those of $S_{1}$ and $T_{1}$. Third, on the basis of the calculated excitation energies of $T_{1}$ and $Q_{1}$ and the experimental ones of $T_{1}$, we shall discuss with which compounds $Q_{1}$ is likely to be accessible through homo-TTA or hetero-TTA. Fourth, we shall investigate how the magnetic high-field effect on the delayed fluorescence is changed when the quintet channel of TTA is also effective. 


\section{Calculation of the excitation energies of the first excited singlet, triplet and quintet states}

\subsection{Computational method and parameters}

The application of ab initio methods to the calculation of multiconfiguration wavefunctions and energies of excited states is limited to small molecules. For molecules larger than benzene. and that means, for most of the molecules of interest here, semi-empirical model hamiltonians have to be used in order to reduce the computational problem to a manageable size. There exist a great variety of semi-empirical methods which differ in the choice of the model hamiltonian and the parameters and in taking into account all valence electrons or $\pi$ electrons only.

In the well-known PPP ${ }^{\dagger}[31-34]$ and $\mathrm{CNDO} / \mathrm{S}^{\div}$ [35-40] variants most often cited in the literature, parameter sets have bcen optimized to yield the best agreement with selected experimental excitation energies. In this sense not only semi-empirical values for certain integrals have to be regarded as parameters, but also the selection procedure for the configurations included in the final configuration interaction calculation. This optimization has been done separately for singlet states [40.41] and for triplet states [42-45], and hence the parameter set is applicable only to the multiplicity for which it has been optimized. Application of a parameter set to the "wrong" multiplicity gives much worse results. On the other hand, the use of different parameters for singlet states and triplet states may lead to inconsistent results, e.g. a singlet state may be calculated lower in energy than its triplet counterpart [46]. It is, therefore, to be expected that neither of the parameter sets will be adequate for quintet-state calculations. A quintet-optimized procedure, however, would be useless since no experimental data on quintet states are available for adjusting the parameters.

This dilemma would not exist which a calculational procedure giving results of comparable ac-

+ Standard abbreviations: PPP: Pariser-Parr-Pople. CNDO: complete neglect of differential overlap. INDO: internediate neglect of differential overlap. S: spectroscopy. $\mathrm{Cl}$ : configuration interaction. MO: molecular orbital. SCF: self-consistent field. curacy both for singlet and triplet energies for in this case it would be justified to expect calculated quintet-state energies to be of about the same accuracy as singlet- and triplet-state energies. A method with these properties has been developed by one of us [47]. Here we restrict ourselves to a description of the essential features of the method.

The model hamiltonian is of the INDO/S type. This allows for the splitting of singlet and triplet levels resulting from $n \rightarrow \pi^{*}$ excitations. In addition. the interaction between ${ }^{2} \pi \pi^{*}$ configurations and ${ }^{3} \sigma \sigma^{*}$ configurations is taken into account - in contrast to CNDO/S where these excitations do not $\operatorname{mix}$ [48]. The parameter $k$ which scales the \%-overlap relative to the $\sigma$-overlap for the calculation of core-hamiltonian matrix elements, and all one-center integrals are taken from the CNDO/S procedure [40]. Electron-electron repulsion integrals are approximated by the Pariser-Parr formula [32.33.49.50].

Energies and wavefunctions of excited states are obtained in a Cl calculation based on groundstate SCF molecular orbitals. In order to have for the three multiplicities $C I$ bases of approximately. equal quality. doubly excited configurations are included also for singlets and triplets. If one takes into account only singly and doubly excited configurations, the ratio of the numbers of configurations to be taken into account in the singlet. triplet and quintet cases. respectively. is $2: 3: 1$. An energy-selected basis of 200 configurations was found to be sufficient in the singlet case in this type of model [51]. Therefore we used 200 singlet. 300 triplet and 100 quintet configurations. respectively: in order to maintain the relation given above.

With the values of the $\beta_{A}$ parameters taken from the CNDO/S procedure [40]. a good correlation was found for singlet and triplet states of a variety of compounds: the calculated excitation energies were $\approx 10 \%$ higher than the experimental ones. This discrepancy was reduced by changing the $\beta_{C}$ parameter by $=10$, from $-17.5 \mathrm{eV}$ to a new value of $-16.0 \mathrm{eV}$.

\subsection{Calculated excitation energies of $S_{1} . T_{1}$ and $Q_{1}$ : comparison with experimenal values}

It is not possible to estimate in advance which class of organic compounds most likely contains 


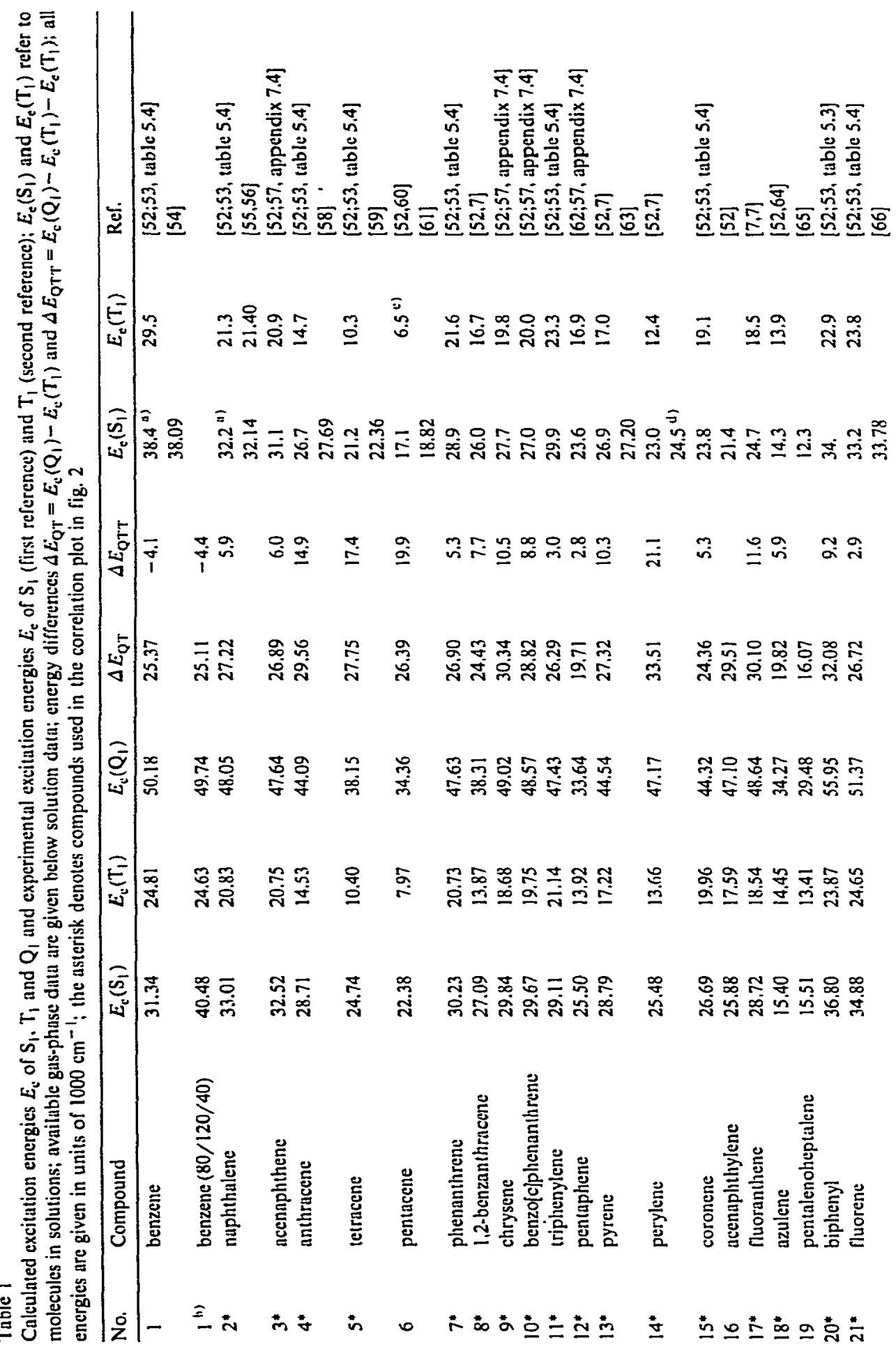



离

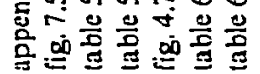

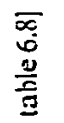

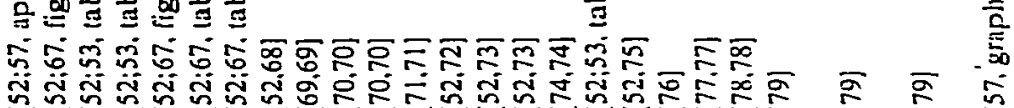

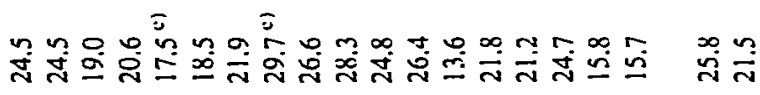

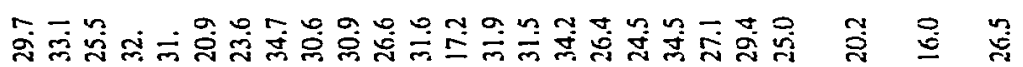

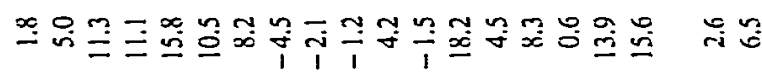

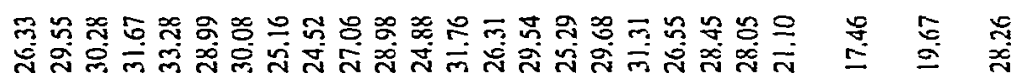

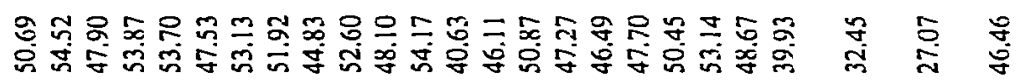

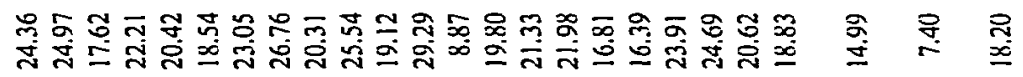

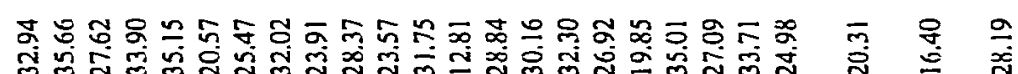

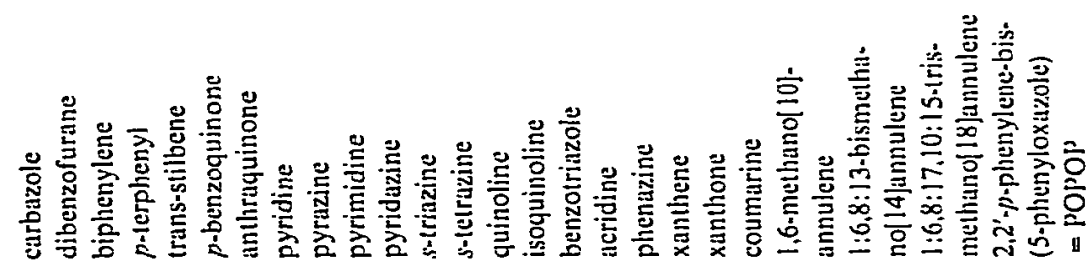

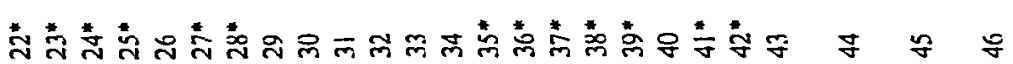




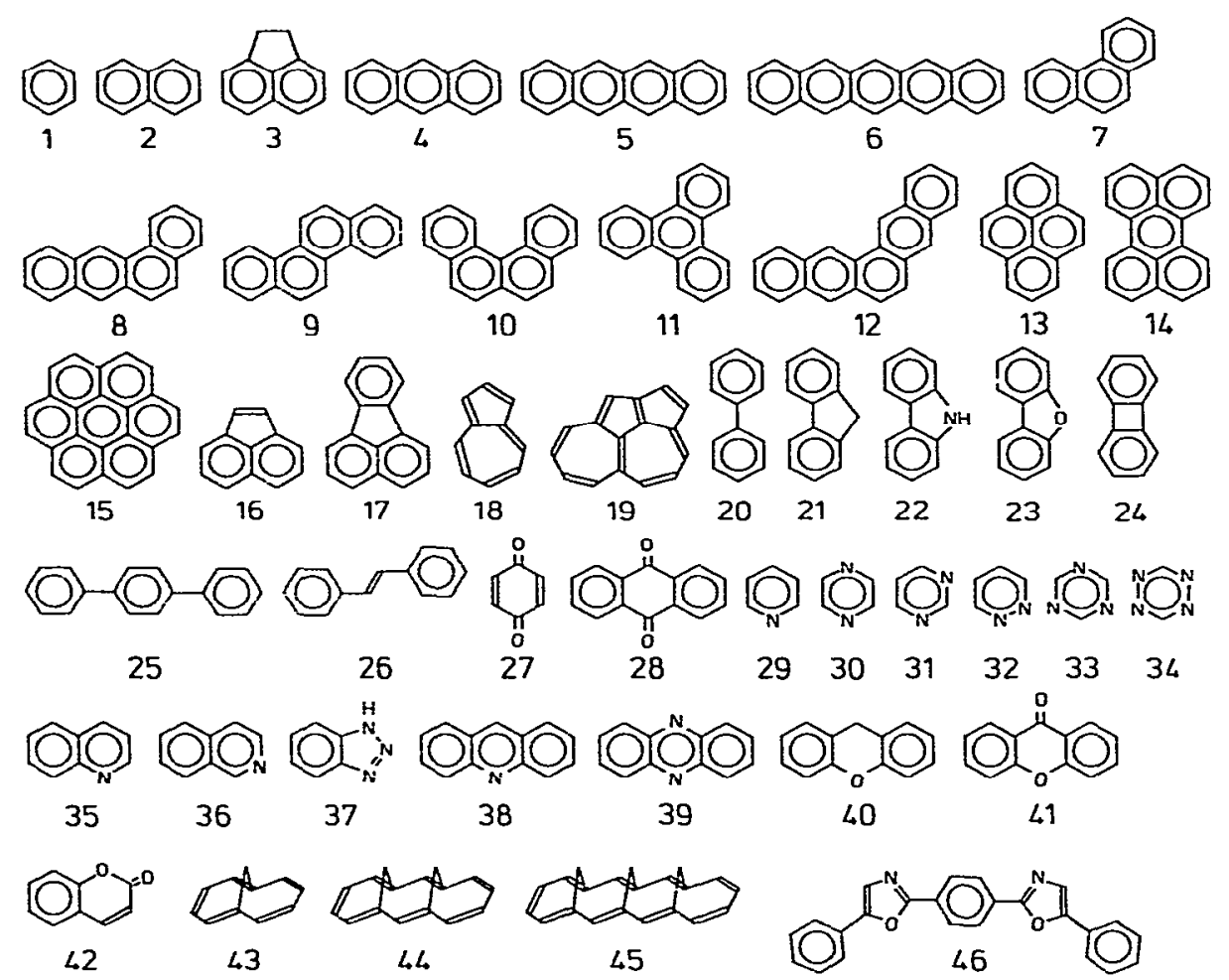

Fig. 1. Structural formulae of the compounds in table 1.

molecules with the property $E\left(\mathrm{Q}_{1}\right) \leqslant 2 E\left(\mathrm{~T}_{1}\right)$. Our choice of compounds for the present calculations was guided in part by experimental considerations (easily observable delayed fluorescence) and in part it was rather arbitrary. In fig. I the structural formulae of the selected compounds are shown, and in table 1 calculated excitation energies of $S_{1}$. $T_{1}$ and $Q_{1}$ are compared with available experimental values.

The calculated excitation energies refer (a) to isolated molecules in the gas phase and (b) to vertical transitions (nuclear distances remain unchanged). Experimental excitation energies refer (a) in most cases to molecules in solution and (b) to 0,0 transitions. Discrepancies between calculated and experimental excitation energies up to a few $1000 \mathrm{~cm}^{-1}$ may result from differences with respect to (a) or (b). An example for (a) is perylene (no. 14): the transition $S_{1} \leftarrow S_{0}$ is strongly allowed, and the red-shift of this transition resulting from solute-solvent interaction is of the order of 2000 $\mathrm{cm}^{-1}$. An example for (b) is fluoranthene (no. 17): the 0,0 transitions of the $S_{1} \leftarrow S_{0}$ absorption band and the $S_{1} \rightarrow S_{0}$ fluorescence band are weak, and the fluorescence exhibits an unusually large Stokes shift of $\approx 4000 \mathrm{~cm}^{-1}[57$, graph $119 \mathrm{C}]$; in this case the vertical-transition energy is expected to be considerably larger than the 0,0-transition energy. (In the phosphorescence spectrum of fluoranthene the 0,0 transition is the strongest band; hence the agreement of the calculated and experimental triplet excitation energies is not surprising.) Triplet excitation energies do not much depend on the solvent.

The calculational error aiso increases somewhat with the size of the molecule due to the fact that, with increasing number of electrons in the molecule, a decreasing fraction of the total number of configurations is taken into account. This is equivalent to a decrease of the energy limit up to which configurations are taken into account.

In general calculated and experimental exci- 


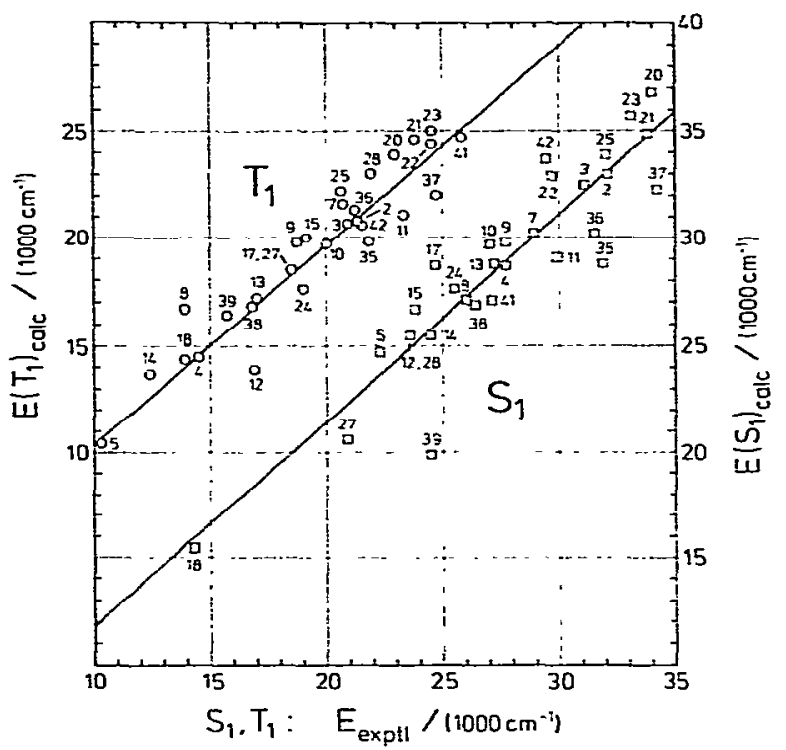

Fig. 2. Correlation plot of the calculated excitation energies $E_{\mathrm{c}}\left(\mathrm{S}_{1}\right)$ and $E_{\mathrm{c}}\left(\mathrm{T}_{1}\right)$ versus the experimental excitation energies $E_{\mathrm{c}}\left(\mathrm{S}_{1}\right)$ and $E_{\mathrm{c}}\left(\mathrm{T}_{1}\right)$. respectively, of the compounds in table 1 . Benzene and the azabenzenes were not included in the correlation plot for reasons given in the text. A few other compounds were also omitted because no reliable values of $E_{c}\left(T_{1}\right)$ were available.

tation energies agree quite well, both for $S_{1}$ and $T_{1}$. With some compounds. however, the agreement is rather poor, the worst cases being benzene (no. 1) and its aza-analogues pyrazine (no. 30). pyridazine (no. 32) and s-tetrazine (no. 34). This fact demands some explanation. It is known that the convergence of transition energies is rather poor for benzene in the CNDO CI scheme [51]. The CI basis of the size applied here accounts already for a large fraction of the correlation energy in the $S_{1}$ state, while the corresponding contribution to the ground state is provided by higher-lying configurations not included. This is exemplified by reduction of the number of configurations to $40 \%$, that is, to 80 singlets, 120 triplets and 40 quintets (table 1, row 2). This removes the doubly excited configurations coupling most strongly to $S_{1}$. (The corresponding energy cut-off criterion is $12.5 \mathrm{eV}$. the same as in the larger basis for naphthalene.) The $T_{1}$ and $Q_{1}$ excitation energies are nearly unaffected by this truncation, in accordance with their generally lower sensitivity to correlation effects (see below). The calculated excitation energies in the first row of table 1 are then best explained by the assumption that the error is made for the ground state which is lacking in $=5000-6000$ $\mathrm{cm}^{-1}$ of correlation energy.

In the case of pyrazine and pyridazine our results can be compared to those of Chen and Hedges [80]. These authors also calculated singlet and triplet excitation energies with a single parameter set in INDO CI. Their parameterization scheme. however. contains much more adjustable parameters than ours. and the parameters were adjusted in order to get best results for six-nembered $\mathrm{N}$-heterocycles. (Note. that in our procedure the change of the $\beta_{C}$ parameter is the only deviation from the CNDO/S standard parameterization.) In spite of this, their calculated excitation energies for pyrazine and pyridazine deviate from the experimental ones by as much as $5000 \mathrm{~cm}^{-1}$. Obviously the excited states of these compounds cannot be properly described in the framework of a semi-empirical method restricted to valence orbital excitation. but diffuse virtual orbitals and Rydberg orbitals are required.

A correlation plot of the calculated excitation energies ( $E_{\mathrm{c}}$ ) against the experimental ones $\left(E_{\mathrm{e}}\right)$ is shown in fig. 2. The correlation lines are $E_{\mathrm{c}}\left(\mathrm{S}_{1}\right)=$ $2140 \mathrm{~cm}^{-1}+0.963 E_{\mathrm{c}}\left(\mathrm{S}_{1}\right)$ and $E_{\mathrm{c}}\left(\mathrm{T}_{1}\right)=1070$ $\mathrm{cm}^{-1}+0.929 E_{\mathrm{e}}\left(\mathrm{T}_{1}\right)$ : the average differences $\Delta E_{\mathrm{ce}}$ $=\left(E_{\mathrm{c}}-E_{\mathrm{c}}\right)_{\lambda 2}$ are $J E_{\mathrm{cc}}\left(\mathrm{S}_{\mathrm{l}}\right)=1130 \mathrm{~cm}^{-1}$ and $\Delta E_{\mathrm{ce}}\left(\mathrm{T}_{1}\right)=-320 \mathrm{~cm}^{-1}$ : the standard deviations $\sigma$ of the $E_{\mathrm{c}}$ values from the regression lines are $\sigma\left(S_{1}\right)=1960 \mathrm{~cm}^{-1}$ and $\sigma\left(T_{1}\right)=1300 \mathrm{~cm}^{-1}$. The deviations of the slopes $m$ of the regression lines from unity are not yet significant. because for both regression lines $(1-m)\left[\left(E_{c}\right)_{m, s}-\left(E_{c}\right)_{m n n}\right]$ is still smaller than the respective standard deviation. The comparatively high value of $\Delta E_{\mathrm{ce}}\left(\mathrm{S}_{1}\right)$ and the higher value of $\sigma\left(S_{1}\right)$ (both relative to the $T_{1}$ correlation) result at least in part from the stronger solvent dependence of the $E_{\mathrm{e}}\left(\mathrm{S}_{1}\right)$ values.

For a number of compounds we have ascertained that our method yields also higher excited singlet and triplet states in the right order and with satisfactory accuracy. As examples we compare calculated and experimental excitation energies of naphthalene in table 2 and of azulene in table 3. As far as reliable assignments of higher 
Table 2

Electronically excited states of naphthalene. Calculated (c) and experimental (gas, sol) excitation energies ( $E$ ) refer to the singlet ground state and are given in units of $1000 \mathrm{~cm}^{-1}$. The symmetry notation (Sym.) is based on $x$-axis $=$ long axis, $y$-axis $=$ short axis and z-axis perpendicular to the molecular plane. Calculated oscillator strengths $f$ refer to $S_{n} \leftarrow S_{0}$ and $T_{n} \leftarrow T_{1}$

\begin{tabular}{|c|c|c|c|c|c|}
\hline No. & Sym. & $E_{\mathrm{c}}$ & $f$ & $E_{\mathrm{gas}}$ & $E_{\mathrm{s}(01}$ \\
\hline 0 & $I^{1} A_{B}$ & 0.00 & & & \\
\hline I & $I^{1} \mathrm{~B}_{3 \mathrm{u}}^{\mathrm{b}}$ & 33.01 & $5 \times 10^{-5}$ & $32.14^{21}$ & $32.2^{(b, h)}$ \\
\hline 2 & $1^{1} B_{2 v}$ & 36.11 & 0.066 & & $36.4^{(b)}$ \\
\hline 3 & $I^{\prime} B_{1 \mathrm{~B}}$ & 42.08 & & & 42.1 (a.d) \\
\hline 4 & $2^{\prime} A_{B}$ & 43.79 & & & $44.5^{\text {c.d) }}$ \\
\hline 5 & $2^{1} B_{2 u}^{b}$ & 47.82 & 0.235 & & \\
\hline 6 & $2^{\prime} B_{3 u}$ & 48.23 & 1.196 & $47.5^{c 1}$ & $45.3^{b)}$ \\
\hline 7 & $3^{1} \mathrm{~A}_{\mathrm{g}}$ & 48.35 & & & $48.8^{c 1}$ \\
\hline 11 & $3^{1} B_{2 w}$ & 53.59 & 0.043 & & $52.5^{\prime \prime}$ \\
\hline 13 & $4^{1} \mathrm{~A}_{\mathrm{g}}^{20}$ & 54.50 & & & $55.7 \mathrm{~g})$ \\
\hline 25 & $4^{1} B_{2 u}^{8}$ & 61.99 & 0.475 & $62.1^{\circ}$ & $59.8^{\prime \prime}$ \\
\hline 1 & $1^{3} B_{2 u}$ & 20.83 & & $21.40^{h)}$ & $21.3^{i 1}$ \\
\hline 2 & $1^{3} B_{3 u}$ & 27.99 & & & $30.8^{i)}$ \\
\hline 7 & $2^{3} \mathrm{~A}_{\mathrm{E}}$ & 41.70 & 0.005 & & $38.7^{\prime \prime}$ \\
\hline 11 & $3^{3} \mathrm{~B}_{18}$ & 46.83 & 0.093 & & $45.5^{\prime \prime}$ \\
\hline 26 & $4^{3} B_{1 B}$ & 59.00 & 0.011 & & \\
\hline 27 & $4^{3} \mathrm{~A}_{\mathrm{g}}$ & 59.08 & 0.057 & & $58.9^{11}$ \\
\hline 34 & $S^{3} B_{1 \mathrm{~g}}$ & 64.83 & 0.158 & & $63.7^{\prime \prime}$ \\
\hline 1 & $1^{5} A_{g}$ & 48.05 & & & \\
\hline 2 & $1^{5} B_{2 u}^{8}$ & 55.61 & & & \\
\hline 3 & $2^{5} A_{g}$ & 58.67 & & & \\
\hline 4 & $1^{5} B_{3 u}$ & 59.24 & & & \\
\hline 5 & $2^{5} B_{2 u}$ & 61.96 & & & \\
\hline
\end{tabular}

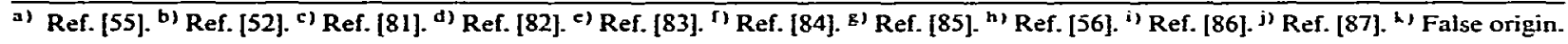

excited states can be made with these compounds. the agreement of calculated and experimental values is good and extends beyond $E_{\mathrm{c}}\left(\mathrm{Q}_{\mathrm{l}}\right)$.

For the assessment of the reliability of the calculated quintet excitation energies, the following observation is important: The contribution of doubly excited configurations to the lowest triplet state was much smaller than thai to the lowest excited singlet state, although the triplet calculation included much more doubly excited configurations than the singlet calculation. Thus the lowest triplet states showed in general only 0.5 to $3.0 \%$ doubly excited character (relative to the ground state), whereas the corresponding singlet states had 3 to $20 \%$ doubly excited character. In the singlet case the inclusion of doubly excited configurations is essential for obtaining the correct order of states, in the triplet case it seems to mainly correct for the depression of the ground state. This is in line with the observation that standard CNDO/S calculations with Pariser integrals give already satisfactory results for the low-lying triplet states $[42,43]$. Of course, doubly excited configurations may have a strong effect on higher triplet states [90].

One can rationalize the different effect of doubly excited configurations on singlet states and triplet states in terms of the different correlation mechanisms involved. Triplet states always have at least two electrons in open shells, and therefore the correlation energy for these electrons is of intershell character. Singlet states, however, though expressed in open-shell configurations, could be of mainly closed-shell character when expressed in their natural orbitals. This involves the intrashell correlation energy, which is known to be larger than the intershell energy $[90,91]$ and which, in the picture of the ground-state $\mathrm{MO} \mathrm{Cl}$ approach, will 
Table 3

Electronically excited states of azulene. Calculated $\left(E_{c}\right)$ and experimental $\left(E_{\mathrm{e}}\right)$ excitation energies refer to the singlet ground state and are given in units of $1000 \mathrm{~cm}^{-1}$. The symmetry notation (Sym.) is based on $z$-axis $=$ in-plane twofold rotation axis. $x$-axis in the molecular plane and $y$-axis perpendicular to it. Calculated oscillator strengths $f$ refer to $S_{n} \leftarrow S_{0} . T_{n} \leftarrow T_{1}$ and $T_{n} \leftarrow T_{2} . E_{e}\left(S_{n}\right)$ refers to azulene in perfluorohexane at room temperature

\begin{tabular}{|c|c|c|c|c|c|}
\hline No. & Sym. & $\overrightarrow{E_{\mathrm{c}}}$ & $E_{c}$ & $f_{n}-0$ & \\
\hline 0 & $l^{1} A_{1}$ & 0.00 & 0.0 & & \\
\hline 1 & $1^{l} B_{1}$ & 15.40 & 14.3 & 0.005 & \\
\hline 2 & $2^{\prime} A_{1}$ & 26.01 & 28.6 & 0.019 & \\
\hline 3 & $2^{1} \mathrm{~B}_{1}$ & 32.51 & 34.0 & 0.036 & \\
\hline 4 & $3^{1} A_{1}$ & 36.02 & 36.4 & 0.358 & \\
\hline 5 & $3^{1} \mathrm{~B}_{1}$ & 38.24 & & 0.001 & \\
\hline 6 & $4^{1} \mathrm{~B}_{1}$ & 42.11 & & 0.036 & \\
\hline 7 & $4^{1} A_{1}$ & 42.16 & 41.3 & 0.295 & \\
\hline 1 & $1^{3} B_{1}$ & 14.45 & $13.9^{\prime 3}$ & $f_{n \leftarrow 1}$ & $f_{n-2}$ \\
\hline 2 & $1^{3} A_{1}$ & 14.91 & & & \\
\hline 3 & $2^{3} A_{1}$ & 19.46 & & 0.0003 & 0.0004 \\
\hline 4 & $2^{3} \mathrm{~B}_{1}$ & 27.23 & & 0.0001 & 0.0000 \\
\hline 5 & $3^{3} B_{1}$ & 32.99 & ? & 0.0322 & 0.0110 \\
\hline 6 & $3^{3} \mathrm{~A}_{1}$ & 34.26 & & 0.0012 & 0.0022 \\
\hline 7 & $4^{3} \mathrm{~B}_{1}$ & 35.94 & & 0.0001 & 0.0011 \\
\hline 8 & $5^{3} \mathrm{~B}_{1}$ & 36.72 & $38^{b . c 1}$ & 0.0019 & 0.0021 \\
\hline 12 & $6^{3} \mathrm{~B}_{1}$ & 42.42 & $41.7^{b . d)}$ & 0.0063 & 0.0100 \\
\hline 16 & $7^{3} A_{1}$ & 48.63 & & 0.0000 & 0.0249 \\
\hline 17 & $7^{3} B_{1}$ & 49.47 & & 0.0299 & 0.0007 \\
\hline 1 & $1^{s} A_{1}$ & 34.27 & & & \\
\hline 2 & $1^{5} B_{1}$ & 44.94 & & & \\
\hline 3 & $2^{5} B_{1}$ & 47.34 & & & \\
\hline 4 & $2^{5} \mathrm{~A}_{1}$ & 48.75 & & & \\
\hline
\end{tabular}

a) 0,0-transition of phosphorescence from azulene in a phena-

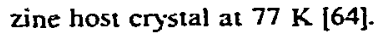

b) Estimated from triplet-triplet absorption [88].

c) Shoulder.

d) Maximum.

C) Not observed [89].

be accounted for by doubly and higher excired configurations. If one restricts the $\mathrm{Cl}$ basis to singly excited configurations. then the correlation effects of the singlet states can be partly accounted for by the choice of the parameters, leading to different parameter sets for singlet and triplet states.

We conclude from these observations that the influence of the higher excited configurations on the lowest excited states decreases with increasing multiplicity. We estimate that the effect of triply excited configurations on the calculated excitation energies of the lowest quintet states would be less than $1 \%$. Therefore we further conclude that the present method yields the excitation energies of the lowest quintet states with about the same accuracy as those of the lowest excited singlet and triplet states.

\subsection{Accessibility of the lowest quintet state by triplet-triplet annihilation}

The energy condition for the population of the lowest quintet state $Q_{1}$ by triplet-triplet annihilation is

$\Delta E=E\left(\mathrm{Q}_{1}\right)-E\left(\mathrm{~T}_{1}\right)-E\left(\mathrm{~T}_{1}^{\prime}\right) \leqslant 0$.

With homo-TTA $T_{1}=T_{1}^{\prime}$ and with hetero-TTA $\mathrm{T}_{1} \approx \mathrm{T}_{1}^{\prime}$. For $E\left(\mathrm{Q}_{1}\right)$ only calculated values are available. For $E\left(\mathrm{~T}_{1}\right)$ and $E\left(\mathrm{~T}_{1}^{\prime}\right)$ in general both. experimental and calculated values are available. Hence. there are three possible ways for the evaluation of eq. (2) in the case of homo-TTA. The best choice is to take for $E\left(T_{1}\right)$ one calculated value, $E_{\mathrm{c}}\left(\mathrm{T}_{1}\right)$. and one experimental value. $E_{\mathrm{c}}\left(\mathrm{T}_{1}\right)$. and to define

$$
\begin{aligned}
\Delta E_{\mathrm{QT}} & =E_{\mathrm{c}}\left(\mathrm{Q}_{1}\right)-E_{\mathrm{c}}\left(\mathrm{T}_{1}\right) \\
\Delta E_{\mathrm{QTT}} & =E_{\mathrm{c}}\left(\mathrm{Q}_{1}\right)-E_{\mathrm{c}}\left(\mathrm{T}_{1}\right)-E_{*}\left(\mathrm{~T}_{1}\right) \\
& \equiv \Delta E_{\mathrm{QT}}-E_{\mathrm{c}}\left(\mathrm{T}_{1}\right) .
\end{aligned}
$$

The evaluation of the quintet-TTA condition according to (4) has two advantages: First. if the calculated excitation energies of $S_{1}$ and $T_{1}$ strongly deviate from the experimental values. this may result from a large systematic error affecting the calculated excitation energies of all states in the same way. Examples for this case are benzene (no. 1). pyrazine (no. 30). and s-tetrazine (no. 34). With these compounds the agreement between the energy differences $E_{\mathrm{c}}\left(\mathrm{S}_{1}\right)-E_{\mathrm{c}}\left(\mathrm{T}_{1}\right)$ and $E_{\mathrm{c}}\left(\mathrm{S}_{1}\right)-$ $E_{\mathrm{c}}\left(\mathrm{T}_{1}\right)$ is much better than that between $E_{\mathrm{c}}\left(\mathrm{S}_{1}\right)$ and $E_{c}\left(\mathrm{~S}_{1}\right)$ or $E_{\mathrm{c}}\left(\mathrm{T}_{1}\right)$ and $E_{\mathrm{c}}\left(\mathrm{T}_{1}\right)$. Hence. by the definition (4) any systematic error affecting $E_{\mathrm{c}}\left(\mathrm{Q}_{1}\right)$ and $E_{\mathrm{c}}\left(\mathrm{T}_{1}\right)$ in the same way is automatically cancelled. Second. in the case of hetero-TTA one would always take the experimental value for $E\left(T_{1}^{\prime}\right)$, and not the calculated one. The definition (4) is consistent with this practice. 
In the following discussion we treat homo-TTA (1) and hetero-TTA (II) separately, and we restrict ourselves to those compounds for which it is known or likely that a delayed fluorescence can be observed.

(I) In table 1 five compounds have negative values of $\Delta E_{\mathrm{QTT}}$ : benzene (no. 1), pyridine (no. 29). pyrazine (no. 30), pyrimidine (no. 31), and s-triazine (no. 33). Of these compounds benzene is of particular interest, because it should be suitable for an experimental investigation, and because our calculation supports the early conjecture of Sternlicht et al. [25] that $Q_{1}$ of benzene might be populated by homo-TTA. The absolute value of $\Delta E_{\mathrm{QTT}}$ for benzene, $4100 \mathrm{~cm}^{-1}$, is still not large enough to predict with certainty that $Q_{1}$ of benzene is accessible through TTA. On the other hand, if we allow for an error in $\Delta E_{\mathrm{QTT}}$ as large as $\pm 4000 \mathrm{~cm}^{-1}$, some other compounds should also be considered as possible candidates for quintetTTA: triphenylene (no. 11), pentaphene (no. 12). fluorene (no. 21). carbazole (no. 22), benzotriazole (no. 37), and xanthone (no. 41). From these examples follows that Suna's [15] general statement of the inaccessibility of the lowest quintet state through homo-TTA is untenable.

For phenanthrene (no. 7), 1,2-benzanthracene (no. 8) and pyrene (no. 13) the $\Delta E_{\mathrm{QTT}}$ values are 5300,7700 and $10300 \mathrm{~cm}^{-1}$, respectively. It is very unlikely that quintet-TTA can take place with one of these compounds, in contrast to the conjecture of Lendi et al. [19]. (In their second theoretical paper on TTA [20], these authors state that the magnetic-field dependence of the delayed fluorescence of these compounds at high temperatures does not yield conclusive evidence for quintetTTA.)

(II) The energy condition for quintet heteroTTA is $\Delta E_{\mathrm{QT}} \leqslant E\left(\mathrm{~T}_{1}^{\prime}\right)$. If we take as second compound benzene with $E\left(T_{1}^{\prime}\right)=29500 \mathrm{~cm}^{-1}$, then table 1 shows that with many compounds $Q_{1}$ should be accessible by hetero-TTA. Even if we allow for a large systematic error in all $\Delta E_{\mathrm{OT}}$ values of table 1, e.g. that all $\Delta E_{\mathrm{OT}}$ values are too small by $4000 \mathrm{~cm}^{-1}$, then still several compounds are left that are suitable for quintet hetero-TTA, e.g. 1,2-benzanthracene (no. 8), pentaphene (no. 12), azulene (no. 18), pentalenoheptalene (no. 19), and the three annulenes (nos. 43-45). Of these compounds at least the first three should be suitable for an experimental investigation (with azulene a delayed fluorescence $S_{2} \rightarrow S_{0}$ resulting from hetero-TTA can be observed [92.93]).

3. Influence of quintet triplet-triplet annihilation on the magnetic-field dependence of the delayed fluorescence

In this section we investigate which magneticfield dependence of the delayed fluorescence should be observed when quintet-TTA is energetically possible and indeed takes place. There are four cases of interest: homo-TTA in molecular crystals, homo-TTA in liquid solutions, heteroTTA in molecular crystals, and hetero-TTA in liquid solutions. Here we only treat the high-field effect in the first, the second, and the fourth case.

\subsection{Homo-triplet-triplet annihilation in molecular crystals}

The theory of the magnetic-field dependence of the delayed fluorescence of pure (undoped) molecular crystals has been developed by Merrifield [5,16], Johnson and Merrifield [14], Suna [15], and Sibani and Pedersen [24]. Here we follow the simpler theory of Johnson and Merrifield [14]. The kinetic model of this theory is shown in the following reaction scheme, which differs from the original one by the explicit inclusion of the quintet channel and by allowing for the population of upper excited states through TTA.

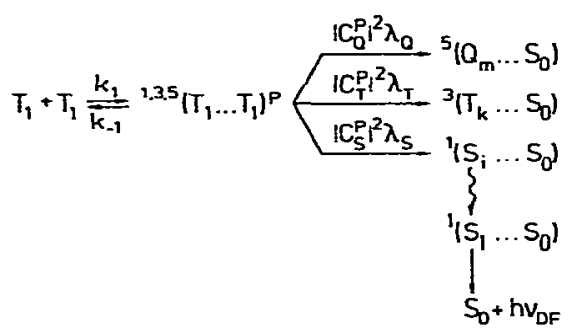

Mobile and uncorrelated triplet excitons $T_{1}$ form 
pairs of correlated triplet excitons in a diffusioncontrolled reaction (second-order rate constant $k_{1}$ ). A triplet pair can either dissociate again (first-order rate constant $k_{-1}$ ) or react. the reaction product being one excited singlet or triplet or quintet state. The creation of singlet excitons $S$, leads to an observable delayed fluorescence (DF). The basic assumptions of the theory are:

(a) The spin-lattice relaxation times are much shorter than the lifetime of the triplet excitons. and the temperature is high (e.g. $\geqslant 100 \mathrm{~K}$ ). Hence. for the present purpose. the concentrations of triplet excitons in the three triplet substates can be assumed to be equal.

(b) The spin-lattice relaxation times are still nuch longer than the lifetime of a triplet pair.

(c) Though triplet interaction is necessary for the correlation of the triplet excitons in a pair. the triplet-interaction energy is assumed to be zero.

(d) There are nine possible pair states ( $p=$ $1,2, \ldots .9)$. The pair states are eigenstates of the pair spin-Hamilton operator, but in general not eigenstates of the total-spin operator. That means. a pair state $(p)$ is in general a singlet-tripletquintet mixture with amplitude factors $C_{S}^{p} . C_{\mathrm{T}}^{p}$, and $C_{O}^{p}$ (referring to the appropriate eigenfunctions of total spin) and $\left|C_{S}^{r}\right|^{2}+\left|C_{\mathrm{T}}^{r}\right|^{2}+\left|C_{\mathrm{Q}}^{r}\right|^{2}=1$.

(e) For a given triplet pair. the effective rate constants for the three TTA channels (5) to (7) are $\left|C_{\mathrm{Q}}^{p}\right|^{2} \lambda_{\mathrm{Q}},\left|C_{\mathrm{T}}^{\rho}\right|^{2} \lambda_{\mathrm{T}}$, and $\left|C_{\mathrm{S}}^{p}\right|^{2} \lambda_{\mathrm{S}}$. respectively, where $\lambda_{\mathrm{Q}}, \lambda_{\mathrm{T}}$, and $\lambda_{\mathrm{S}}$ are multiplicity-specific first-order rate constants.

The magnetic-field dependence of TTA. and hence of the observable delayed fluorescence. results from the interplay between spin-spin interaction in the individual triplet excitons (characterized by the zero-field splitting parameters $D$ and $E$ ) and Zeeman interaction on the one hand. and from the relative magnitudes of $k_{-1}, \lambda_{Q}, \lambda_{T}$. and $\lambda_{S}$ on the other hand.

The second-order rate constant $k_{\mathrm{s}}$ for the total singlet-TTA takes a particularly simple form in zero magnetic field and in the high-field limit. The basic equation for $k_{\mathrm{s}}$ is the same in both cases and follows directly from the reaction scheme (5) $-(7)$ :

$$
k_{\mathrm{S}}=\frac{1}{9} k_{1} \sum_{p=1}^{9} \frac{\left|C_{S}^{p}\right|^{2} \lambda_{\mathrm{S}}}{k_{-1}+\left|C_{S}^{p^{2}}\right|^{2} \lambda_{\mathrm{S}}+\left|C_{\mathrm{T}}^{p}\right|^{2} \lambda_{\mathrm{T}}+\left|C_{\mathrm{Q}}^{p}\right|^{2} \lambda_{\mathrm{Q}}} .
$$

In zero field and in the high-ficld limit, the pair states are either singlet-quintet mixtures or pure quintet states or pure triplet states [14]. In zero field there are three pair states with singlet character. each with $1 / 3$ singlet and $2 / 3$ quintet character. Hence from (9) follows

$k_{S}(0)=\frac{1}{9} k_{1} \times 3 \frac{\frac{1}{3} \lambda_{S}}{k_{-1}+\frac{1}{3} \lambda_{S}+\frac{3}{3} \lambda_{0}}$.

In the high-field limit. with the magnetic field in off-resonance direction (see below). there are only. two pair states with singlet character. one with $1 / 3$ singlet and $2 / 3$ quintet character. and one with $2 / 3$ singlet and $1 / 3$ quintet character [14]. Hence from (9) follow's

$$
\begin{aligned}
& k_{S}(x)=\frac{1}{4} k_{1} \\
& \quad \times\left(\frac{\frac{1}{3} \lambda_{S}}{k_{-1}+\frac{1}{3} \lambda_{S}+\frac{2}{3} \lambda_{0}}+\frac{k_{-1}+\frac{\frac{2}{3}}{\frac{2}{3}} \lambda_{S}+\frac{1}{3} \lambda_{Q}}{k_{0}}\right) .
\end{aligned}
$$

(With the magnetic field in resonance direction. the two pair states with singlet character are degenerate and split into a pure singlet pair state and a pure quintet pair state [14].)

At low triplet exciton concentrations. most triplet excitons decay by other processes than TTA. The intensity $I_{\mathrm{DF}}$ of the observable delayed fluorescence is then proportional to $k_{\mathrm{s}}$. and the relative change of $I_{\mathrm{DF}}$ in the high-field limit is

$$
\begin{aligned}
\frac{I_{\mathrm{DF}}(x)-I_{\mathrm{DF}}(0)}{I_{\mathrm{DF}}(0)} & \approx \frac{k_{S}(x)-k_{S}(0)}{k_{S}(0)} \\
& =\frac{-2\left(\lambda_{S}-\lambda_{Q}\right)}{3\left(3 k_{-1}+2 \lambda_{S}+\lambda_{Q}\right)} .
\end{aligned}
$$

Obviously, a positive high-field effecr would imply $\lambda_{\mathrm{Q}}>\lambda_{\mathrm{S}}$ and hence would mean that quintet-TTA takes place. (In an actual experimental investigation it would be necessary to ascertain that TTA is the only magnetic-field sensitive process, because other magnetic-field sensitive processes. for exam- 
ple quenching of triplet excitons by free radicals [94], might also lead to a positive high-field effect.)

\subsection{Homo-triplet-triplet annihilation in fluid solutions}

Theoretical models for the magnetic-field dependence of the delayed fluorescence of liquid solutions of aromatic compounds were developed by Avakian et al. [17], Atkins and Evans [18], and Lendi et al. $[19,20]$. Here we follow the perturbation-theoretical approach of Lendi et al. [20]. in which the possibility of quintet-TTA is already included, and which leads to closed formulae in the limit of low viscosity (where rotational relaxation of the interacting triplet molecules can be assumed to be much faster than TTA). According to ref. [20], eq. (3.31). the relative change of $I_{D F}$-in the high-field limit is given by

$$
\begin{aligned}
& \frac{I_{\mathrm{DF}}(\infty)-I_{\mathrm{DF}}(0)}{I_{\mathrm{DE}}(0)} \approx \frac{-64\left(D^{*}\right)^{2}}{45\left(\lambda_{\mathrm{S}}+k_{-1}\right)\left(\lambda_{\mathrm{Q}}+k_{-1}\right)} \\
& \quad \times \frac{\left(\lambda_{\mathrm{S}}-\lambda_{\mathrm{Q}}\right)\left(\lambda_{\mathrm{S}}+\lambda_{\mathrm{Q}}+2 k_{-1}\right)}{\left[\left(\lambda_{\mathrm{S}}+\lambda_{\mathrm{Q}}+2 k_{-1}\right)^{2}+4 J_{\mathrm{Q}}^{2}\right]}
\end{aligned}
$$

where $\lambda_{\mathrm{S}}, \lambda_{\mathrm{Q}}$ and $k_{-1}$ have the same meaning as before; $J_{\mathrm{Q}}$ is the singlet-quintet splitting of the triplet pair in zero field, and $D^{*}$ is defined by

$D^{*}=\left(D^{2}+3 E^{2}\right)^{1 / 2}$,

with $D$ and $E$ the zero-field splitting parameters of the triplet state. Again, as in the case of homo-TTA in molecular crystals, a positive high-field effect would imply that quintet-TTA takes place.

\subsection{Hetero-triplet-triplet annihilation in fluid solutions}

The magnetic-field dependence of hetero-TTA in molecular crystals has been investigated experimentally and theoretically $[95,96]$. Examples of delayed fluorescence resulting from hetero-TTA in liquid solutions are known (from $S_{1}$ [97] and from upper excited singlet states $[92,98])$. However, the magnetic-field dependence of hetero-TTA in liquid solutions has not yet been investigated, neither experimentally nor theoretically. In the appendix we show that the perturbation-theoretical approach of Lendi.et al. [20] can be easily adapted to the case of hetero-TTA. Eq. (13) remains valid in the case of hetero-TTA, if for $\left(D^{*}\right)^{2}$ the arithmetic mean is taken for the two triplet states $\left(T_{1}\right)_{1}$ and $\left(T_{1}\right)_{2}$,

$$
\left(D^{*}\right)^{2}=\left[\left(D_{1}^{*}\right)^{2}+\left(D_{2}^{*}\right)^{2}\right] / 2 \text {. }
$$

Hence the qualitative conclusion to be drawn from a positive high-field effect would be the same as in the case of homo-TTA.

\subsection{Discussion}

A general theory of TTA has to tackle three problems: first, to calculate for a triplet pair with definite relative orientation and distance and definite pair spin state the probabilities of transitions to all possible final states $[25,99.100]$ (final-states problem); second, to treat the effect of an external magnetic field [5,13-24] (spin problem); third. to average over all possible relative orientations and distances and over all final states (averaging problem). In all theories of the magnetic-field dependence of TTA [5,13-24] the tacit assumption is made that it is possible to separate the final-states problem from the spin problem and to treat it phenomenologically by introducing the spinspecific rate constants $\lambda_{\mathrm{S}}, \lambda_{\mathrm{T}}$ and $\lambda_{\mathrm{Q}}$. In the case of molecular crystals this assumption is justified when TTA is essentially restricted to nearest neighbours and only one relative orientation of molecules has to be taken into account. In the case of liquid solutions it is questionable whether the separation of the final-states problem from the spin problem can be justified in the general theory of ref. [19]. Lendi et al. [19] simply state that they treat the magnetic-field dependence of TTA as a problem in spin space alone, without giving any justification. We believe that at least one has to be very cautious in deriving any conclusions from the quantizative agreement between theory and experiment in the case of liquid solutions with high viscosity.

If we assume that the theories in sections $3.1-3.3$ 
are essentially correct, then the qualitative proof of quintet-TTA will depend on the existence of compounds (homo-TTA) or pairs of compounds (hetcro-TTA) with the property $\lambda_{\mathrm{Q}} / \lambda_{\mathrm{S}}>1$. One may assume that the variation of $\lambda_{Q} / \lambda_{S}$ (when quintetTTA is energetically possible) should be ahout the same as that of $\lambda_{S} / \lambda_{T}$. When quintet-TTA cannot take place, the ratio $\lambda_{\mathrm{S}} / \lambda_{\mathrm{T}}$ is closely related to the excited-singlet yield $q_{\mathrm{S}}$ in TTA. From the fact [13]. that in liquid solutions under similar experimental conditions $q_{\mathrm{S}} \approx 0.56$ for naphthalene and $q_{\mathrm{s}} \approx 0.08$ for anthracene. one may conclude that in general each of the three rate constants $\lambda_{\mathrm{S}}, \lambda_{\mathrm{T}}$ and $\lambda_{\mathrm{Q}}$ will substantially depend on the initial triplet states and on the accessible final excited states of the respective multiplicity. Moreover, since in the case of liquid solutions the $\lambda$ are average values with respect to relative orientation and distance, one should expect a greater variation of the $\lambda$ in the case of molecular crystals. Hence there is some hope that systems with the property $\lambda_{Q} / \lambda_{S}>1$ can be found.

If $\lambda_{\mathrm{Q}}$ is smaller than $\lambda_{\mathrm{S}}$. but still of the same order of magnitude, then one can try to prove $\lambda_{\mathrm{Q}}>\mathbf{0}$ by a quantitative evaluation of the observed magnetic-field dependence of the delayed fluorescence. However, at least in the case of liquid solutions [20] there is, apart from the necessary caution mentioned above, the additional problem that the theory contains only three independent combinations of the five parameters $k_{-1}, \lambda_{\mathrm{S}}, \lambda_{\mathrm{Q}}$, $J_{\mathrm{Q}}$ and $D^{*}$. $D^{*}$ can be obtained from an independent ESR experiment. $k_{-1}$ cannot be measured independently. One can only estimate the order of magnitude of $k_{-1}$, and one may expect that in the small viscosity range, in which the theory [20] is valid, $k_{-1}$ is the only temperature- and viscositydependent parameter and has the temperature dependence of a diffusion coefficient, that is $k_{-1} \propto$ $T / \eta$ ( $T$ is the temperature and $\eta$ is the viscosity).

\section{Summary and conclusions}

(1) Using an INDO CI procedure including singly and doubly excited configurations, we have calculated the excitation energies of the lowest excited singlet states $\left(S_{1}\right)$. triplet states $\left(T_{1}\right)$ and quintet states $\left(Q_{1}\right)$ for forty-six conjugated organic compounds.

(2) With most compounds calculated values $\left(E_{\mathrm{c}}\right)$ and experimental values $\left(E_{\mathrm{e}}\right)$ of the excitation energies of $S_{1}$ and $T_{1}$ agree satisfactorily. Within the error limits of the method, the correlation of $E_{\mathrm{c}}$-values with $E_{\mathrm{c}}$-values is about the same for $\mathrm{S}_{1}$ and $T_{1}$.

(3) With compounds where the unambiguous assignment of upper excited states is possible (naphthalene, azulene), the satisfactory agreement between calculated and experimental values of singlet and triplet states extends beyond $E_{\mathrm{c}}\left(\mathrm{Q}_{1}\right)$.

(4) From (2) and (3) and from the consideration that triply and higher excited configurations should only little contribute to $E_{\mathrm{c}}\left(\mathrm{Q}_{1}\right)$. we conclude that our INDO $\mathrm{Cl}$ procedure yields $E\left(\mathrm{Q}_{3}\right)$ with about the same accuracy as $E\left(\mathrm{~S}_{1}\right)$ and $E\left(\mathrm{~T}_{1}\right)$.

(5) On the basis of the calculated excitation energies of $Q_{1}$ and $T_{3}$ and the known experimental excitation energies of $T_{1}$. we conclude that $Q_{1}$ of benzene is likely to be accessible through homoTTA, and that $Q_{1}$ of many compounds should be accessible through hetero-TTA.

(6) If quintet-TTA takes place, and if for the multiplicity-specific rate constants $\lambda_{s} . \lambda_{0}$ the relation $\lambda_{\mathrm{Q}}>\lambda_{\mathrm{S}}$ holds. then a positive magnetic highfield effect on the intensity of the delaved fluorescence should be observed with homo-TTA in molecular crystals and with homo- and hetero-TTA in liquid solutions of low viscosity.

\section{Acknowledgement}

BD gratefully acknowledges a research fellowship of the Deutsche Forschungsgemeinschaft and thanks Professor G. Hohlneicher for support. BN thanks Dr. J. Wirz for his interest in the quintetTTA problem and for helpful HMO calculations in an early stage of this investigation. We thank the Regionales Rechenzentrum Köln for providing the necessary computer time. 
Appendix: Modification of the Lendi-GerberLabhart (LGL) theory for the case of hetero-triplet-triplet annihilation

We use the same nomenclature as LGL in their papers I [19] and II [20] and also refer to their formula numbers. To incorporate the case of hetero-TTA we have to consider two sets of zero-field splitting parameters, $D_{1} . E_{1}$ and $D_{2}, E_{2}$. The triplet-triplet interaction hamiltonian $\mathscr{K}^{(1)}(11,2.7)$ then takes the form

$$
\begin{aligned}
& \mathcal{K}^{(1)}\left(\Omega_{1}, \Omega_{2}\right) \\
& =\sum_{i=1}^{2} \sum_{m=-2}^{2} \sum_{n=-1}^{1} g_{2 n}(i) \mathcal{Q}_{m 2 n}^{(2)}\left(\Omega_{i}\right) T_{2}^{m}(i) .
\end{aligned}
$$

The only difference between (A.1) and (II, 2.7) is that $g_{2 n}$ in (II, 2.7) is replaced by $g_{2 n}(i)$ in (A.1) with

$g_{0}(i)=D_{i} / 3$

and

$g_{2}(i)=g_{-2}(i)=6^{-1 / 2} E_{i}, \quad i=1,2$

In the perturbational treatment of the magneticfield dependence of TTA [20], the only matrix elements required are $\mathcal{K}_{1 j}^{(1)}(5 \leqslant l \leqslant 9)$, whose absolute square $(11,3.21)$

$f^{(\prime)}\left(\Omega_{1}, \Omega_{2}\right)=\left|\mathcal{K}_{1 /}^{(1)}\left(\Omega_{1}, \Omega_{2}\right)\right|^{2}, \quad 5 \leqslant l \leqslant 9$,

has to be isotropically averaged:

$$
\begin{aligned}
f_{0}^{(1)}= & \overline{\left|\mathcal{C}_{1 /}^{(1)}\left(\Omega_{1}, \Omega_{2}\right)\right|^{2}} \\
= & \sum_{i, k=1}^{2} \sum_{m, m^{\prime}=-2}^{2} \sum_{n, n^{\prime}=-1}^{1}\left[T_{2}^{m}(i)\right]_{1} \\
& \times\left[T_{2}^{m^{\prime}}(k)\right]_{n} g_{2 n}(i) g_{2 n^{\prime}}(k) \\
& \times \overline{\mathcal{P}_{m 2 n}^{(2)}\left(\Omega_{i}\right) \mathcal{Q}_{m^{2} 2 n^{\prime}}^{(2)}\left(\Omega_{k}\right)} .
\end{aligned}
$$

According to eqs. (II, A3.1) and (II, A3.2)

$$
\begin{aligned}
& \overline{\mathcal{Q}_{m^{\prime} \cdot m}^{(j)}(\Omega)}=\delta_{j 0} \delta_{m 0} \delta_{m^{\prime} 0}, \\
& \overline{\mathcal{P}_{m_{1}^{\prime} m_{1}}^{\left(j_{1}\right)}(\Omega) \mathscr{Q}_{m_{2}^{\prime} m_{2}}^{\left(j_{j}\right)}(\Omega)}
\end{aligned}
$$

$$
=\delta_{j_{2} j_{2}} \delta_{-m_{1} m_{2}} \delta_{-m_{1} m_{2}} \frac{(-1)^{m_{1}-m_{1}}}{2 j_{1}+1}
$$

(A.5) and (A.6) imply that in eq. (A.4) all terms with $i \neq k$ vanish. Therefore

$$
\begin{aligned}
f_{0}^{(i)}= & \sum_{i=1}^{2} \sum_{m=-2}^{2} \sum_{n=-1}^{1}\left[T_{2}^{m}(i)\right]_{1}\left[\left(T_{2}^{-m}(i)\right]_{n}\right. \\
& \times g_{2 n}(i) g_{-2 n}(i)(-1)^{m} / 5 .
\end{aligned}
$$

Taking the matrix elements of $T_{2}^{m}$ from table A4.1 in ref. [19] and taking into account (A.2), we finally get

$$
\begin{aligned}
f_{0}^{(\prime)} & =\frac{2}{5} \sum_{i=1}^{2}\left[g_{0}^{2}(i)+2 g_{2}^{2}(i)\right] \\
& =\frac{2}{45}\left(D_{1}^{2}+D_{2}^{2}+3 E_{1}^{2}+3 E_{2}^{2}\right)=\frac{4}{45}\left(D^{*}\right)^{2} .
\end{aligned}
$$

Therefore, the extension of the LGLII theory to the case of the hetero-TTA only requires the substitution of $D^{2}$ and $E^{2}$ by the average values $\left(D_{1}^{2}+D_{2}^{2}\right) / 2$ and $\left(E_{1}^{2}+E_{2}^{2}\right) / 2$.

\section{References}

[1] K. Itoh, Chem. Phys. Letters 1 (1967) 235.

[2] R.A. Huber. M. Schwoerer. H. Benk and H. Sixl, Chem. Phys. Letters 78 (1981) 416.

[3] H. Benk and H. SixI. Mol. Phys. 42 (1981) 779.

[4] C. Kollmar. H. Sixl, H. Benk, V. Denner and G. Mahler, Chem. Phys. Letters 87 (1982) 266.

[5] R.E. Merrifield, J. Chem. Phys. 48 (1968) 4318.

[6] S. Czannecki, Bull. Acad. Polon. Sci. Ser. Sci. Math. Astron. Phys. 9 (1961) 561.

[7] B. Nickel, Helv. Chim. Acta 61 (1978) 221.

[8] C.A. Parker, C.G. Hatchard and T. Joyce, J. Mol. Spectry. 14 (1964) 311.

[9] K. Kikuchi, H. Kokubun and M. Koizumi. Bull. Chem. Soc. Japan 41 (1968) 1545.

[10] R.P. Groff, R.E. Merrifield and P. Avakian, Chem. Phys. Letters 5 (1970) 168.

[11] J.B. Birks, Chem. Phys. Letters 7 (1970) 293.

[12] D. Wyrsch and H. Labhart, Chem. Phys. Letters 12 (1971) 373.

[13] F. Tfibel and L. Lindqvist, Chem. Phys. 10 (1975) 471

[14] R.C. Johnson and R.E. Merrifield, Phys. Rev. B1 (1970) 896.

[15] A. Suna, Phys. Rev. B1 (1970) 1716.

[16] R.E. Merrifield, Pure Appl. Chem. 27 (1971) 481.

[17] P. Avakian, R.P. Groff, R.E. Kellog, R.E. Merrifield and A. Suna, in: Organic scintillators and liquid scintillation 
counting, eds. D.L. Horrocks and C.T. Peng (Academic Press. New York. 1971) p. 499.

[18] P.W. Atkins and G.T. Evans. Mol. Phys. 29 (I975) 921.

[19] K. Lendi. P. Gerber and H. Labhart. Chem. Phys. 18 (1976) 449.

[20] K. Lendi. P. Gerber and H. Labhart, Chem. Phys. 20 (1977) 145 .

[21] C.E. Swenberg and N.E Geacintov, in: Organic molecular photophysics. Vol. 1, ed. J.B. Birks (Wiley, New York. 1973) ch. 10.

[22] N.E. Geacintov and C.E. Swenberg. in: Luminescence spectroscopy, ed. M.D. Lumb (Acadenic Press. New York. 1978) ch. 4.

[23] M. Pope and C.E. Swenberg. Electronic processes in organic crystals (Wiley. New York. 1982).

[24] P. Sibani and J.B. Pedersen. Phys. Rev. B26 (1982) 2584.

[25] H. Sternlicht. G.C. Nieman and G.W. Robinson. J. Chem. Phys. 38 (1963) 1326.

[26] R.G. Parr, D.P. Craig and I.G. Ross. J. Chem. Phys. Is (1950) 1561

[27] J. Spichtig. H. Bulska and H. Labhart. Chem. Phys. 15 (1976) 279

[28] P.B. Empedocles and J.W. Linnett. Proc. Roy. Soc. A282 (1964) 166.

[29] S.D. Peyerimhoff and R. Buenker. Theoret. Chim. Acta 19 (1970) 1.

[30] J. Wirz. private communication.

[31] J.A. Pople. Trans. Faraday Soc. 44 (1953) 1375.

[32] R.G. Parr and R. Pariser. J. Chem. Phys. 21 (1953) 466.

[33] R.G. Parr and R. Pariser, J. Chem. Phys. 21 (1953) 767.

[34] R. Pariser. J. Chem. Phys. 24 (1956) 250.

[35] J. Del Bene and H.H. Jaffe. J. Chem. Phys. 48 (1968) 1807.

[36] J. Del Bene and H.H. Jaffe. J. Chem. Phys. 48 (1968) 4050.

[37] J. Del Bene and H.H.Jaffe, J. Chem. Phys. 49 (1968) 1221.

[38] J. Del Bene and H.H.Jaffe, J. Chem. Phys. 50 (1969) 1126.

[39] R.L. Ellis and H.H. Jaffe, J. Mol. Spectry. 50 (1974) 474.

[40] R.L. Ellis, G. Kuehnlenz and H.H. Jaffe. Theoret. Chim. Acta 26 (1972) 131.

[41] J. Pancir and R. Zahradnik. J. Phys. Chem. 77 (1973) 121.

[42] H.M. Chang and H.H. Jaffe, Chem. Phys. Letters 23 (1973) 146.

[43] C.A. Masmanidis. H.H. Jaffe and R.L. Ellis. J. Phys. Chem. 79 (1975) 2052.

[44] J. Pancir and R. Zahradnik. J. Phys. Chem. 77 (1973) 107.

[45] J. Pancir and R. Zahradnik. J. Phys. Chem. 77 (1973) 114.

[46] R.W. Bigelow, H.-J. Freund and B. Dick. Theoret. Chim. Acta, submitted for publication.

[47] B. Dick. to be published.

[48] T. Shibuya and T. Funada. Chem. Phys. Letters 70 (19S0) 199.
[49] R.G. Parr. J. Chem. Phys. 20 (1952) 1499

[50] R. Pariser. J. Chem. Phys. 21 (1953) 568.

[51] B. Dick and G. Hohlneicher. Theoret. Chim. Acta 53 (1979) 221.

[52] UV atlas of organic compounds (Butterworths. London/Verlag Chemie. Weinhein. 1966-1971).

[53] J.B. Birks. Photophrsics of aromatic molecules (Wiley-Interscience. New York. 1970).

[54] G. Herzberg. Mfolecular spectra and molecular structurc. Vol. 3. Electronic spectra and electronic structure of polyatomic molecules (Van Nostrand. Princeton. 1967) p. 556.

[55] M. Stockburger. H. Gattermann and W. Klusmann. J. Chem. Phys. 63 (1975) 4529.

[56] H. Gattermann and M. Stockburger. J. Chem. Phys. 63 (1975) 4541.

[57] I.B. Berlman. llandbook of fluorescence spectra of aromatic molecules, 2nd Ed. (Academic Press, Niew York. 1971).

[58] T.R. Hays, W. Henke. H.L. Selzle and E.W. Schlag. Chem. Phys. Letters 77 (1981) 19.

[59] A. Amirav: U. Fven and J. Intner. Chem. Phys 51 (1980) 31.

[60] N.E. Geacintov. J. Burgos. M. Pope and C. Strom. Chem. Phys. Letters 11 (1971) 504.

[61] A. Amiras: U. Even and J. Jortner, Chem. Phys. Letuers $71(1980) 1$.

[62] E. Clar. Polycyclic hydrowarbons. Vol. 1 (Academic Press. New York. 1964) p. 357.

[63] N. Ernsting. private communication

[64] D. Klemp and B. Nickel. Chem. Phys. 78 (19S3) 17

[65] K. Haffner. R. Fleischer and K. Friz. Angew. Chem. 77 (1965) 42.

[66] A. Amirav. U. Even and J. Jortner. J. Chem. Phys. 75 (19\$1) 3151 .

[67] S.P. McGlynn. T. Azumi and M. Kinoshita. Molecular spectroscopy of the triplet state (Prentice-Hall. Englewood Cliffs. 1969).

[68] D.F. Evans. J. Chem. Soc. (1957) 3855.

[69] A. Rousset. J. Chim. Phys. 61 (196-1) 1621.

[70] B.J. Cohen and L. Goodman. J. Chem. Phys. 46 (1967) 713.

[71] L. Goodman. J. Mol. Spectry: 6 (1961) 109.

[72] J.R. McDonald and L.E. Brus, J. Chem. Phys. 59 (1973) 4966.

[73] F. Dörr and H. Gropper. Ber. Bunsenges. Physik. Chem. 67 (1963) 193.

[74] H.U. Schütt and H. Zimmermann. Ber. Bunsenges. Physik. Chem. 67 (1963) 54.

[75] B. Nickel and H.-J. Karbach. unpublished resulis.

[76] V. Zanker and E. Ehrhardt. Buil. Chem. Soc. Japan 39 (1966) 1694.

[77] B. Nickel and G. Roden. Chem. Phys. Letters 74 (1950) 368

[78] E.T. Harrigan. A. Chakrabarti and $\mathrm{N}$. Hirota. J. An. Chem. Sec. 95 (1976) 3460.

[79] H.J. Dewey. H. Deger, W. Frölich, B. Dick, K.A. Klin- 
gensmith, G. Hohlneicher, E. Vogel and J. Michl. J. Am. Chem. Soc. 102 (1980) 6412.

[80] S.-Y. Chen and R.M. Hedges, Theoret. Chim. Acta 31 (1973) 275.

[81] B. Dick and G. Hohlneicher, Chem. Phys. Letters 84 (1981) 471 .

[82] N. Mikami and N. Ito. Chem. Phys. Letters 31 (1975) 472.

[83] E.E. Koch. A. Otto and K. Radler. Chem. Phys. Letters 16 (1972) 131.

[84] H.B. Klevens and J.R. Platt. J. Chem. Phys. 17 (1949) 470.

[85] D. Bebelaar, Chem. Phys. 3 (1974) 205.

[86] D.M. Hanson and G.W. Robinson, J. Chem. Phys. 43 (1963) 4174.

[87] Y.H. Meyer, R. Astier and J.M. Leclercq. J. Chem. Phys. 56 (1972) 801.
[88] H. Görner and D. Schulte-Frohlinde. J. Photochem. 16 (1981) 169.

[89] R. Görner, private communication.

[90] M.K. Orloff, J. Chem. Phys. 47 (1967) 235.

[91] O. Sinanoglu: J. Chem. Phys. 36 (1962) 706.

[92] B. Nickel. Chem. Phys. Letters 68 (1979) 17.

[93] H.-J. Kray and B. Nickel. Chem. Phys. 53 (1980) 235.

[94] V. Ern and R.E. Merrifield. Phys. Rev. Letters 21 (1968) 609.

[95] R.P. Groff, R.E. Merrifield, P. Avakian and Y. Tomkiewicz. Phys. Rev. Letters 25 (1970) 105.

[96] M. Chabr and I. Zschokke-Gränacher. J. Chem. Phys. 64 (1976) 3903.

[97] C.A. Parker. Proc. Roy. Soc. A276 (1963) 125.

[98] B. Nickel and G. Roden. Chem. Phys. 66 (1982) 365.

[99] J. Jortner. S.A. Rice and J. Katz, J. Chem. Phys. 42 (1965) 309.

[100] C.E. Swenberg. J. Chem. Phys. 51 (1969) 1753. 